Notfall Rettungsmed 2013 · 16:5-7

DOI 10.1007/s10049-013-1690-1

Online publiziert: 14. Februar 2013

(c) Springer-Verlag Berlin Heidelberg 2013
U. Kreimeier ${ }^{1} \cdot$ H.-R. Arntz ${ }^{2}$

${ }^{1}$ Klinik für Anaesthesiologie, Klinikum der Universität München

2 Medizinische Klinik II, Kardiologie und Pulmologie, Charité -

Universitätsmedizin Berlin, Campus Benjamin Franklin, Berlin

\title{
Wissenschaftliche Evidenz: Dokumentation originärer Ergebnisse
}

\author{
Ein Plädoyer zur Publikation \\ in wissenschaftlichen Zeitschriften
}

Der Prozess für die Überarbeitung der ILCOR-Leitlinien [1] ist im Dezember in die nächste Stufe getreten: Nachdem die Arbeitsgruppen gebildet wurden, ist nunmehr zu identifizieren, welche neuen wissenschaftlichen Erkenntnisse vorliegen, die berücksichtigt werden und Eingang in die Arbeitsblätter („worksheets“) finden und dann unter den Fachleuten hinsichtlich Qualität und Aussagekraft diskutiert und bewertet werden sowie welcher Evidenzgrad ihnen zugeordnet wird.

Mehr als 50 Jahre moderne Herz-Lungen-Wiederbelebung, da stellt sich schon die Frage: Was wissen wir und warum sind wir uns bei einigen Fragstellungen in der Beantwortung einfach noch nicht so sicher? Als Beispiele sind aktuell die Diskussion um den Zeitpunkt des Beginns der induzierten kontrollierten Hypothermie (bereits in der präklinischen Phase?) oder die Konzeption der Telefonreanimation durch Leitstellendisponenten zu nennen - Letztere erstmals verankert in den ILCOR-Leitlinien zur Reanimation 2010 und nunmehr verbunden mit der Diskussion, wie sie am besten und effektivsten durchzuführen sei.

Ein Blick in die Literaturdatenbanken zeigt uns den Weg, welche Arbeiten (potenziell) neue Erkenntnisse beinhalten. PubMed und Medline sind neben Embase und der Cochrane Library bekannte Datenbanken für Zeitschriften, die den internationalen Standards genügen, um gelistet zu werden und dementsprechend als Quelle zu dienen. Wir veröffentlichen in dieser Aus- gabe von Notfall + Rettungsmedizin einen Beitrag über den plötzlichen Herztod (Arntz), indem gleich im ersten Abschnitt die Definition für den sog. „Plötzlichen Herztod“ gegeben wird. Sie stammt natürlich aus einem englischsprachigen Journal, Verzeihung: aus einer englischsprachigen „Zeitschrift“. Verfolgt der aufmerksame Leser die Herkunft der Definition, landet er schließlich bei einem Buchkapitel aus dem Jahr 1997, aus dem vermutlich eine erste Definition stammt. Hier stellt sich dann neben der korrekten, in diesem Fall aktuellen Definition eines Terminus die Frage, wer denn nun eigentlich ursprünglich die Definition für den „Plötzlichen Herztod“ veröffentlicht hat. Immerhin haben sich so bedeutende Register wie MONICA (Augsburg) daran orientiert.

\section{Wer entdeckte eigentlich Amerika?}

Damit kommen wir zu den Urhebern, den Entdeckern von neuen (Forschungs-)Gebieten. Durch Auftauchen einer antiken Landkarte aus dem Jahr 1418 erhielt vor einigen Jahren das Gerücht Nahrung, dass bereits Jahrzehnte vor der Entdeckung Amerikas durch Christoph Kolumbus der chinesische Seefahrer Zheng He mit seiner Flotte auf diesem Kontinent gelandet sei [4].

Aber wir müssen gar nicht so weit zurückgehen: Wer führte den ersten bemannten Motorflug durch? Dieser - eigent- lich trivialen - Frage ging kürzlich ein Referent im Rahmen der Weiterbildung bei der Bayerischen Landesärztekammer nach, als er über „Evidenz“ sprach. Am 18. August 1901 wurde im Bridgeport Herald über einen erfolgreichen bemannten Motorflug von Gustav Weißkopf am 14. August 1901 in Fairfield/CT über eine Strecke von $800 \mathrm{~m}$ berichtet [5]. Erst 2 Jahre später konnten die Brüder Wright ein ähnliches Flugexperiment erfolgreich präsentieren, welches - wie wir natürlich alle wissen - Eingang in die Weltgeschichte als erster Flug mit einem motorbetriebenen Fluggerät fand. Der Grund, warum man Weißkopfs Leistung nicht entsprechend in der Geschichte honorierte, lag wohl darin, dass keine „Dokumentation“"vorlag, die Gebrüder Wright dagegen einen Fotographen engagierten, der von diesem ersten Flug ein Foto für die Nachwelt machte [6].

\section{In God we trust. All others must bring data [2]. Nur was publiziert wird, zählt!}

Immer wieder kommt es vor, dass man eine interessante Veröffentlichung liest und denkt: „Das ist auch meine Erfahrung." $\mathrm{Ab}$ und zu gerät man dann ins Grübeln, ob man das nicht auch hätte veröffentlichen und so zum Ruhm für die Nachwelt hätte beitragen können. Allerdings werden an wissenschaftliche(s) Arbeiten Ansprüche gestellt, die nicht ganz trivial sind und die auch Berechti- 
Infobox 1

1. Frau Prof. Julie R. Ingelfinger, MD, ist Professorin für Pädiatrie an der Harvard Medical School, Senior Consultantin Pädiatrischer Nephrologie am Massachusetts General Hospital - Mass General Hospital for Children und Deputy Editor des The New England Journal of Medicine (NEJM). Daneben ist Professor Ingelfinger Mitherausgeberin von Kidney International und gehörte dem Herausgeberboard verschiedener Zeitschriften an, so Pediatrics, American Journal of Physiology, Pediatric Nephrology und Hypertension. (Quelle: http://www.uam.es/otros/rccdr/docs/ mexico/Ingelfinger/Bio.Blurblngelfinger. pdf?ID=16844, Zugriff am 12.1.2013)

2. Frau PD Dr. sc. hum. Christel Weiß ist Diplom-Mathematikerin und Leiterin der Abteilung für Medizinische Statistik, Biomathematik und Informationsverarbeitung an der Ruprecht-Karls-Universität Heidelberg. Sie ist u.a. Herausgeberin des Springer-Lehrbuchs „Basiswissen Medizinische Statistik". Sie gehört dem Editorial Board von Notfall + Rettungsmedizin an und fungiert als medizinisch-statistische Beraterin.

3. Prof. em. Dr. med. Hans-Richard Arntz ist Kardiologe und war bis zu seiner Emeritierung im vergangenen Jahr leitender Oberarzt an der Charité - Universitätsmedizin Berlin, Abteilung für Kardiologie und Pneumologie Campus Benjamin Franklin Berlin. Er ist u. a. Herausgeber des Springer-Lehrbuchs „Notfallbehandlung des akutes Koronarsyndroms", Mitglied des Editorial Board von Notfall + Rettungsmedizin seit 1997 und Co-Editor-in-Chief. Er leitete die Working Group „Acute coronary syndrome" im ILCOR Leitlinien-Prozess 2005 und ist Principal Author der ERC-Leitlinien zum ACS 2005 und 2010.

4. Prof. Dr. med. Uwe Kreimeier ist Anaesthesist, Notfallmediziner und Intensivmediziner. Er ist seit 2007 Editor-in-Chief von Notfall + Rettungsmedizin und seit 2008 Section Editor des Scandinavian Journal of Trauma, Resuscitation and Emergency Medicine. Er ist u. a. Mitglied der World Association of Medical Editors (WAME).

gung haben: Schlussfolgerungen („conclusion of the authors") müssen auf Ergebnissen („results“) beruhen, die in der Zusammenschau mit den Ergebnissen anderer bewertet werden müssen („discussion“). Dies wiederum setzt eine adäquate, geeignete Methodik voraus („material and methods"), die Daten liefert, welche mit geeigneten statistischen Verfahren („statistics“) hinsichtlich des Zutref- fens der Nullhypothese überprüft werden. Die wissenschaftliche Fragestellung („problem“) wird also mit validen $\mathrm{Me}$ thoden wissenschaftlich bearbeitet. Die Vergangenheit hat gezeigt, dass ein wissenschaftliches Fehlverhalten („scientific misconduct") im Nachhinein sowohl für Einzelaussagen bis hin zu Ergebnissen von Metaanalysen, aber auch für den Autor selbst Konsequenzen hat.

\section{Was folgt hieraus?}

Hieraus folgt als erstes natürlich, dass nur das gilt, was dokumentiert wird. Dies muss nicht unbedingt in einer hochrangigen Zeitschrift publiziert worden sein, es genügt, ein nachprüfbares (Bild-)Dokument. Aber eine Aussage ist natürlich leichter nachprüfbar, wenn sie „nachlesbar" ist, und sie wird häufiger zitiert, je populärer das Medium ist. Eine Publikation z. B. in einer Zeitschrift wie dem The New England Journal of Medicine mit einem Impactfactor von über 53 erscheint dem „normalen“ Wissenschaftler unerreichbar. Allerdings gibt es auch im Board von Notfall + Rettungsmedizin durchaus Autoren, die dies geschafft haben. Respekt!

\section{Reading between the lines - zwischen den Zeilen...}

Julie Ingelfinger (• Infobox, 1), Boston, Deputy Editor of The New England Journal of Medicine, berichtete Mitte Januar in München, dass die Ablehnquote bei Einreichung von Manuskripten für ihre Zeitschrift über $90 \%$ liegt. Dieses auf den ersten Eindruck völlig entmutigende Faktum muss unseren Blick aber „nicht weg vom Forschen lenken“, sondern hin zur Auswahl der „richtigen Zeitschrift“, oder korrekter, der Zeitschrift mit dem für die dargestellte Untersuchung und die daraus folgenden Ergebnisse richtigen Interessensgebiet, dem Ziel („scope“) der Zeitschrift. Dieses Ziel orientiert sich an dem Gründungsgedanken für eine Zeitschrift, an wissenschaftlichen Schwerpunkten und nicht zuletzt auch an der Leserschaft der Zeitschrift. Potenzielle Autoren durchforsten („screenen“) verschiedene Fachzeitschriften nach Veröffentlichungen, die dem geplanten Manuskript ähnlich sind.
Was macht es auch für einen Sinn, einer pädiatrischen medizinischen Fachzeitschrift ein Manuskript über ein Konzept zur ganzheitlichen Versorgung in der Geriatrie einzureichen...

\section{Der Impact Factor ist nicht alles...}

Das The New England Journal of Medicine hatte 2011 einen Impact Factor von 53,298, Notfall + Rettungsmedizin hat einen Impact Factor (2011) von 0,542. Es ist (k)eine simple mathematische Rechnung zu überlegen, wieviele Manuskripte man publizieren müsste, um einen kumulierten Impact Factor zu erreichen, der einer Veröffentlichung in The New England Journal of Medicine entspricht: ca. 100. Wenn Sie, verehrte Leserinnen und Leser, diesen Gedanken weiterspinnen, dann kommen Sie möglicherweise zu der Erkenntnis, dass Sie mit diesen 100 Manuskripten auch unsere Zeitschrift bedienen könnten...

Voraussetzung für dieses Zahlenspiel ist allerdings, dass die Annahmequote identisch ist. Ein Herausgeber ist gut beraten, wenn er statistische Expertise sucht: Wissenschaftlich wurde die Nullhypothese formuliert, die Wahrscheinlichkeit, dass ein Manuskript zur Publikation angenommen wird, sei gleich der Wahrscheinlichkeit, dass es zurückgewiesen wird.

Die Rücksprache mit Frau PD Dr. Weiß (- Infobox, 2), Mannheim, aus unserem Herausgeberboard führte dazu, dass die Nullhypothese verworfen und die Alternativhypothese („Die Wahrscheinlichkeit, dass ein Beitrag angenommen wird, ist ungleich 0,5.“) angenommen wurde. Dieses Ergebnis allerdings „macht weitere Untersuchungen notwendig" - eine nicht selten im Fazit von wissenschaftlichen Originalarbeiten $\mathrm{zu}$ findende Aussage. Das wachsende nationale und internationale Renommée von Notfall + Rettungsmedizin und die immer größer werdende Zahl an eingereichten Manuskripten hatte 2012 insgesamt (Ersteinreichung und Revisionen) eine Ablehnquote von etwas über $30 \%$ zur Folge.

Sehr geehrte Leserinnen und Leser, Nur durch Weitergabe von neuen Erkenntnissen wächst der Horizont des medizinischen Gesamtwissens. Die Beurteilung jeder einzelnen Beobachtung und je- 
des Gesamtergebnisses einer Studie muss nach wissenschaftlichen Kriterien erfolgen. Eine wissenschaftliche Publikation ist dann interessant, wenn sie von der breiten Gruppe („scientific community“) wahrgenommen wird. Mit Notfall + Rettungsmedizin halten Sie eine Zeitschrift in Händen, deren Herausgeberboard Ihnen interessante, richtungsweisende und originäre Beiträge präsentiert - und das 8-mal im Jahr. Nutzen Sie die Gelegenheit, sich zu informieren, nutzen Sie aber auch die Möglichkeit, selbst über Ihre Erfahrungen $\mathrm{zu}$ berichten und ein wissenschaftliches Manuskript einzureichen.

\section{Mit den besten Grüßen}

Ihre

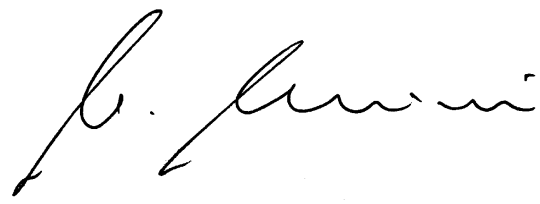

U. Kreimeier

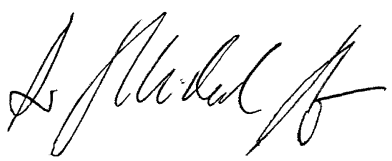

H.-R. Arntz

\section{Korrespondenzadresse}

\section{Prof. Dr. U. Kreimeier}

Klinik für Anaesthesiologie,

Klinikum der Universität München

Nussbaumstraße 20, 80336 München

Uwe.Kreimeier@med.uni-muenchen.de

\section{Compliance with Ethics Guidelines}

Conflict of interest. H.R. Arntz and U. Kreimeie declare that they have no conflict of interest.

This article does not contain any studies with human or animal subjects.

\section{Literatur}

1. ILCOR: International Liaison Committee on Resuscitation. In diesem Editorial werden im Folgenden einige (wenige) englischsprachige Ausdrücke benutzt, die als Fachtermini auch in deutschen Texten Verbreitung gefunden haben

2. "In God we trust. All others must bring data." Möglicherweise fälschlich W.A. Deming (US-amerikanischer Physiker, Statistiker sowie Pionier im Bereich des Qualitätsmanagements) zugeschriebenes Zitat. Quelle: http://en.wikipedia.org/wiki/W._Edwards_Deming, Zugriff am 12.01.2013

3. Ingelfinger J. Reading between the lines. Vortrag am 11. Januar 2013 in der von Haunerschen Kinderklinik des Klinikums der Universität München

4. http://www.stern.de/wissen/natur/antike-karteals-beweis-entdeckten-chinesen-amerika-553361. html

5. http://de.wikipedia.org/wiki/Gustav-Weißkopf

6. http://en.wikipedia.org/wiki/Wright_brothers

\section{Maske statt Tubus}

Wenn die Lunge versagt, gibt es bisher nur eine intensivmedizinische Therapiemöglichkeit - die künstliche, invasive Beatmung über einen Tubus. Neue Erkenntnisse zeigen, dass beim akuten Lungenversagen die Funktion der Lunge durch extrakorporale Verfahren wie die künstliche Lunge unterstützt und sogar komplett ersetzt werden kann. Durch diese Systeme wird dem Blut des Patienten Sauerstoff zugeführt und Kohlendioxid entfernt, dadurch werden Teile der Lungenfunktion übernommen und die Lunge entlastet. Kernstück der künstlichen Lunge ist eine spezielle Membran, die den Austausch von Sauerstoff und Kohlendioxid im Blut ermöglicht. In der Regel wird die künstliche Lunge durch Pumpensysteme angetrieben, um einen größtmöglichen Anteil des Herzminutenvolumens über die Membran zu leiten. Bei einigen Patienten kann mit dieser künstlichen Lunge eine Beatmung sogar ganz vermieden werden.

Eine Intubation hingegen versuchen die Ärzte heute weitestgehend zu vermeiden oder die Intubationszeit kurz zu halten. Bei dieser Form der künstlichen Beatmung kann es nach wenigen Tagen zu Infektionen kommen. Bei schwer kranken Personen ist eine künstliche Beatmung mit einem Tubus allerdings häufig nicht zu verhindern.

Quelle: Deutsche Interdisziplinäre Vereinigung für Intensiv- und Notfallmedizin, www.divi-org.de 